\title{
Situação constrangedora envolvendo profissionais das Unidades Básicas de Saúde do município de Porto Velho/Rondônia
}

Recebido em: 22/10/2010

Aceito em: 21/03/2011
Flávio Salomão Miranda ${ }^{1}$

Daniele Ferreira Garcia² Maria Rita Soares do Nascimento Barreto ${ }^{3}$

Este estudo tem por objetivo estimar as situações constrangedoras envolvendo profissionais das Unidades Básicas de Saúde do município de Porto Velho/Rondônia, caracterizar o fenômeno da violência contra os profissionais de saúde no local de trabalho, abordar aspectos relativos à violência e suas implicações na área de saúde e conhecer os fatores considerados importantes no que tange à violência contra profissionais de saúde. Os dados coletados com médicos, enfermeiros e dentistas, mediante estudo transversal, apontaram que há relevância de aproximação das áreas de educação e de saúde, eixos fundamentais para uma melhor possibilidade de enfrentamento da problemática da violência.

Descritores: Violência, Promoção de Saúde, Prevenção.

\section{Embarrassing involving professional Basic Health Units of the city of Old Port/Rondônia}

This study aims to estimate the embarrassing situations involving professionals from primary care units in the city of Porto Velho, Rondônia, to characterize the phenomenon of violence against health professionals in the workplace, addressing issues related to violence and its implications on health and determining the factors considered important when it comes to violence against health professionals. The data collected by health professionals, doctors, nurses and dentists, by means of transversal study showed that there is relevance to approximate the areas of education and health, the cornerstones for a better chance of coping with problems of violence.

Descriptors: Violence, Health Promotion, Prevention.

\section{Situación embarazosa con profesionales de las Unidades Básicas de Salud del município de Puerto Viejo/Rondônia}

Esse estúdio tiene como objetivo estimar las situaciones embarazosas de profesionales de unidades de atención primaria en la ciudad de Porto Velho, Rondônia, para caracterizar el fenómeno de la violencia contra los profesionales de la salud en el lugar de trabajo, abordando temas relacionados con la violencia y sus consecuencias en la salud y la determinación de los factores considerados importantes cuando se trata de la violencia contra los profesionales de la salud. Los datos recogidos por esses profesionales como médicos, enfermeras y dentistas, por medio de estudio transversal mostró que es pertinente la aproximación de las áreas de educación y salud, factores fundamentales para una mejor oportunidad de hacer frente a los problemas de la violencia.

Descriptores: Violencia, Promoción de la Salud, Prevención.

\section{INTRODUÇÃO}

A violência sempre existiu entre os homens, assim como a cada local e tempo existiram mecanismos sociais para a compreensão e o controle ${ }^{(1)}$. Seguindo esse curso de pensamento, não seria o caso de buscar, no passado, os precursores históricos da violência na contemporaneidade como se houvesse uma linearidade evolutiva que, ao ser conhecida, pudesse ser interrompida, devolvendo toda a inocência e tranquilidade originais, ou a paz, enquanto estado absoluto?

Sabe-se que a violência faz parte da humanidade, mas a presença na vida contemporânea ganha novas proporções, tais como o ódio manifestado em atos violentos e palavras com carga de agressividade, gerando medo que ora aparece sob a forma de resignação, ora de angústia. Ressalta-se ser a violência uma das intensas preocupações em escala mundial, atingindo a sociedade como um todo, grupos ou famílias e, ainda, o indivíduo de maneira isolada. Fazendo parte da chamada questão social, assim revela formas de dominação e opressão desencadeadoras de conflitos. Como um fenômeno complicado e polêmico, a violência é praticada por indivíduos, manifestando-se de várias maneiras, assumindo formas próprias de relações pessoais, sociais, políticas ou culturais ${ }^{(2,3)}$. 
A partir dessas considerações, questiona-se: quais os profissionais de saúde são mais afetados por esse fenômeno? Como estabelecer diretrizes nacionais e locais de prevenção contra a violência?

A violência contra os profissionais de saúde acarreta consequências em níveis individual, institucional e social. É um problema que afeta gravemente a saúde física e mental dos profissionais que são diretamente atingidos, sobretudo dos que testemunham os episódios de violência, prejudicando o desempenho profissional e a qualidade dos cuidados que as unidades de saúde atingidas prestam aos cidadãos ${ }^{(4)}$.

A violência no local de trabalho é, portanto, uma ameaça ao bom funcionamento do setor da saúde. Por tal motivo, abordar um tema como esse torna-se relevante quando inúmeros fatores indicam as desigualdades sociais em limites intoleráveis, estilos de vida, crenças e escolhas pessoais(5).

Nesse contexto, o presente estudo tem como objetivo estimar as situações constrangedoras envolvendo profissionais de três Unidades Básicas de Saúde do município de Porto Velho/Rondônia, bem como caracterizar o fenômeno da violência no local de trabalho, abordar aspectos relativos à violência e suas implicações na área de saúde e conhecer os fatores considerados importantes no que tange a violência contra profissionais de saúde.

\section{METODOLOGIA}

Estudo de abordagem quantitativa no que se refere à população e amostragem. Realizou-se estudo transversal com amostra de 132 profissionais de três Unidades Básicas de Saúde (UBS) do município de Porto Velho/Rondônia. Entre os profissionais que participaram da pesquisa, havia médicos $(n=52)$, enfermeiros $(n=50)$ e dentistas $(n=30)$.

A coleta de dados foi realizada por meio de um questionário utilizado como instrumento composto de perguntas, sendo 22 fechadas e duas abertas. Os dados foram tabulados e submetidos à análise descritiva.

Atenta-se, portanto, ao que preconiza a Resolução n 196/96 do Conselho Nacional de Saúde - Ministério da Saúde, que regulamenta a pesquisa envolvendo seres humanos, sendo respeitados os aspectos éticos e legais, onde foram incorporados os principais princípios éticos: a beneficência, o respeito à dignidade humana (autonomia), a justiça e a equidade.

Assim, os participantes assinaram o Termo de Consentimento Livre Esclarecido-TCLE, no qual foimantido sigilo dos informantes sob os dados pessoais, como nome do(a) entrevistado(a). Esse, por sua vez, poderia interromper o processo de pesquisa a qualquer momento. O trabalho foi autorizado pelo Centro de Estudo de Pessoal (CEP) da Faculdade Integradas Aparício Carvalho, com o número de protocolo 202/2010.

\section{RESULTADOS E DISCUSSÃO}

Entre os 132 participantes, tivemos 58\% $(n=77)$ do gênero feminino e $42 \%$ ( $n=55)$ do gênero masculino, com idades entre 18 e 59 anos, sendo a maior frequência de faixa etária no intervalo de 25 a 39 anos. No que se refere ao tempo de trabalho nas Unidades Básica de Saúde, observou-se um intervalo entre cinco e dez anos, o que evidencia que esses profissionais já possuem experiência na função. Quanto ao atendimento por gênero, não foi observada diferença quanto ao sexo dos pacientes.

Pode-se observar, no gráfico 1, a escala de preocupação com valores de 0 a 10, na qual o 0 significa sem preocupação e 10, muito preocupado.

Ográfico 1 representa dados de todos os profissionais envolvidos na pesquisa, demonstrando que $54 \%(n=71)$ apresentaram um grau maior ou igual a 7, o que evidencia que as trocas sociais, a reciprocidade e a solidariedade, que formam os alicerces das relações sociais, ficam cada vez mais prejudicadas. Percebe-se um aumento da intolerância e uma crescente diminuição das possibilidades de resolução de conflitos de forma dialógica(7).

Observa-se, ainda, que o tipo de violência mais praticado é a ameaça/agressão verbal (praticada por pacientes e/ou familiares de pacientes): $83 \%$. Entende-se por ameaça/agressão verbal um comportamento que humilha, degrada ou indica falta de respeito à dignidade e valor de uma pessoa. É a comunicação por palavras, tom ou postura que rebaixa, ameaça, acusa ou desrespeita o outro.

A pesquisa aponta que 109 de 132 profissionais de saúde (83\%) já sofreram ameaça/agressão verbal, sendo em maior escala enfermeiros, pois, de 50 colaboradores, 45 (90\%) afirmaram sofrer esse tipo de violência. Tal dado evidencia um índice preocupante, o que faz suscitar um questionamento: como estabelecer diretrizes nacionais e locais de prevenção contra a violência?

Em 2001, foi publicada a Política Nacional de Redução da Morbimortalidade por Acidentes e Violências, estabelecida pela Portaria $n^{\circ}$ 737/GM de 16 de maio de 2001, visando a estabelecer diretrizes e estratégias para a redução da morbimortalidade por violência, queenvolvea promoção da adoção decomportamentos e ambientes seguros e saudáveis ${ }^{(7,8)}$.

A política possui princípios da promoção da saúde e destaca a importância do "fortalecimento da capacidade dos indivíduos, das comunidades e da sociedade em geral para desenvolver, melhorar e manter condições e estilos de vida saudáveis. Esse fortalecimento inclui a criação de ambientes saudáveis, a reorganização dos serviços de saúde, o reforço da ação comunitária e o desenvolvimento de aptidões pessoais"(8).

Um aspecto preocupante refere-se à disponibilidade e diligência no setor de saúde para realizar as capacitações de recursos humanos necessárias para a garantia do enfrentamento da questão da violência de forma sensível, humanizada e não estigmatizante. A complexidade do enfrentamento das questões da violência exige grau diferenciado de reflexão e preparo dos trabalhadores da saúde, na medida em que, contrário aos processos saúde-doença usuais. Nesse caso, "cuidadores" e "cuidados" estão envolvidos em um fenômeno compartilhado, podendo alternar papéis entre vítimas e vitimizadores ${ }^{(9)}$.

Assim, se os trabalhadores da saúde pretendem ressaltar a prevenção da violência e a construção da cultura da paz, surge a necessidade de aprendizado em lidar com a resolução de conflitos e ampliar a capacidade de comunicação e diálogo. Lidar com a diferença, manifestar solidariedade, tolerância e respeito, prover um cuidado atento e acolhedor e aceitar o direito à 
Gráfico 1 - Escala de preocupação com atitudes violentas que possam ser praticadas por parte de pacientes e/ou familiares de pacientes na Unidade Básica de Saúde
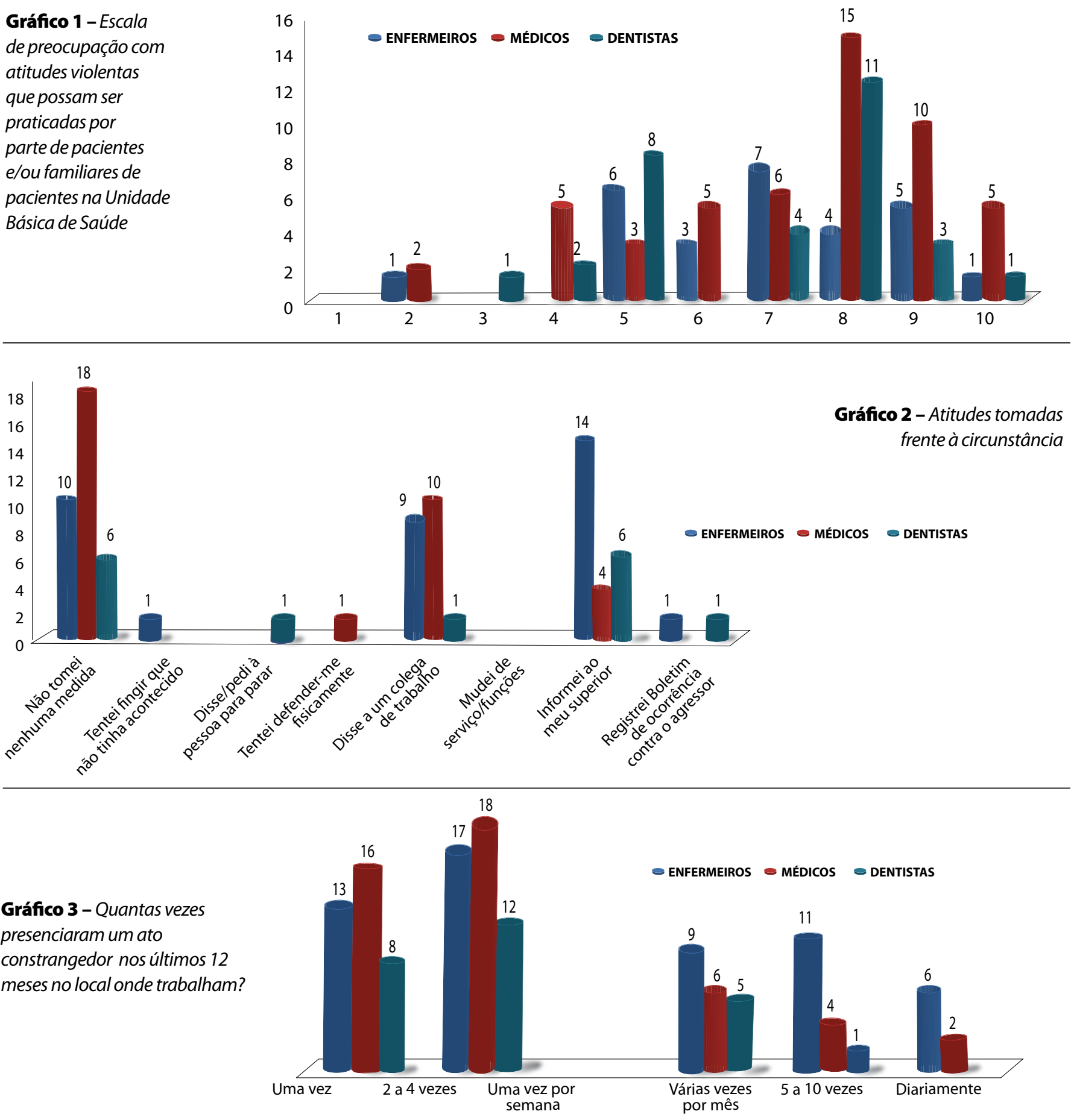

autodeterminação do sujeito sobre sua vida não são qualidades frequentemente disponíveis no setor de saúde.

\section{Reação incidente da violência física}

Entende-se por violência física o uso de força física contra ou grupo que resulta em dano físico, sexual ou psicológico. Inclui bater, esbofetear, esfaquear, alvejar, empurrar, morder, beliscar, contaminar com produtos orgânicos (como, por exemplo, saliva e sangue), entre outros ${ }^{(10)}$. Como pode ser observado no gráfico 2 , os enfermeiros formam o grupo que mais informou o acontecido a seu superior: $26,9 \%$. A maioria dos médicos prefere não tomar nenhuma medida (36\%); entre os dentistas: não houve diferença entre os que informaram ao superior ou os que não tomaram providências.

Obviamente, existem conceitos e até informações acerca de como e por que acontece a violência. Entretanto, a riqueza de muitas explicações sobre o que é violência, bem como as diversas maneiras que se tem de entendê-la, pode tornar confusa a clareza indiciadora de seus fatos. Assim, o desconhecimento de alta prevalência por parte dos profissionais mantém a violência invisível para os serviços de saúde ${ }^{(11,12)}$. 
A violência não costuma ser considerada um problema de saúde pública nem um problema médico típico, e somente a partir da década de 1980, ou seja, recentemente, a violência foi incorporada na agenda da saúde púbica, tanto no Brasil quanto no mundo ${ }^{(13)}$. De acordo com Morgado(14), a violência constituise como um fenômeno de saúde pública "porque afeta a saúde individual e coletiva", exigindo, "para sua prevenção e tratamento, a formulação de políticas específicas e organização de práticas e de serviços particulares ao setor".

A pesquisa permitiu igualmente conhecer a quantidade de vezes que os profissionais presenciaram um ato de violência física em seu local de trabalho nos últimos 12 meses.

Conforme mostra o gráfico 3, 96,9\% dos participantes presenciaram um ato constrangedor (violência física) no serviço. Logo, discute-se os motivos das omissões das denúncias de agressão/violência verbal. Notou-se que $21,9 \%$ dos profissionais $(\mathrm{n}=29)$ que não fizeram a denúncia tiveram medo das consequências. Além disso, $17 \%$ dos profissionais afirmaram que não tiveram tempo para realizá-la. Levando em conta todos esses dados apresentados, pode-se dizer que, ao refletir sobre o processo de globalização que atinge todos os níveis sociais, encontra-se a necessidade de análise de como os profissionais da saúde estão exercendo o trabalho.

Portanto, percebe-se a seriedade de ações decisivas frente à realidade diária dos diversos tipos de violência para a reversão desse problema de saúde coletiva estabelecido. Compete aos profissionais, em especial cuidadores na área da saúde, ter presentes na atividade assistencial, além do papel de cuidador, o de educador, oferecendo outro(s) modelo(s) de relacionamento(15).

É função primordial incentivar a população a repudiar a violência em qualquer oportunidade, em todos os momentos e contatos possíveis, divulgando aspectos desse ideal de proteção aos direitos humanos, orientando, por meio de palestras, especialmente para grupos, criando programas educativos para os que estão em situação de risco, como, por exemplo, os dependentes químicos, pais jovens, incluindo os adolescentes, a população marginalizada pela sociedade, entre outros.

Os meios de comunicação de massa, ao divulgarem informações e reportarem temas relacionados, deveriam contar com o auxílio de profissionais das áreas de saúde e da educação, de forma a melhor repassarem o ideal de proteção integral ao ser humano. Compete a todos participar da organização de grupos multidisciplinares, com estudos e enfrentamento de situações, cada vez mais preparados e instrumentalizados. Masetto ${ }^{(16)}$ afirma que o avanço científico e tecnológico impõe-se na sociedade contemporânea, levando à compreensão da necessidade da formação permanente, criando espaços de mudança de mentalidade acadêmica, ética, política e outras relações com a sociedade, para que essa se torne justa e solidária.

\section{CONCLUSÃO}

O presente trabalho permitiu contextualizar a violência presente em larga escala na sociedade brasileira, legitimando uma forma de poder estruturante nas relações sociais e na interação com fatores pessoais, econômicos e culturais. Cabe a fundamental participação ativa dos profissionais da saúde na atuação e enfrentamento da problemática em questão.

É inegável a presença da violência no ambiente de trabalho dos profissionais de saúde. Políticas de saúde do trabalhador devem aproximar a sociedade do profissional, a fim de estabelecer uma melhoria na relação das partes em questões. Para isso, deve-se incentivar pesquisas nesse sentido, visando a recolher propostas e sugestões dos que realmente sofrem atos constrangedores em seu ambiente de trabalho.

\section{Referências}

1. Zaluar A. Da revolta ao crime S.A. São Paulo: Moderna; 1996.

2. Ferrari DCA, Vecina TCC. O fim do silêncio na violência familiar. Teoria e prática. São Paulo: Agora; 2002.

3. Bicudo HP. Violência: o Brasil cruel sem maquiagem. 4a ed. São Paulo:

Moderna; 1992.

4. Antunes AR, Biscaia A, Conceição C, Fronteira I. Violência contra profissionais de saúde no local de trabalho. Relatório Resumido. V Conferência Psicologia nos Cuidados de Saúde Primários - Psicologia, promoção da saúde e prevenção. 2004.

5. Deslandes SF. Violência no cotidiano dos serviços de emergência hospitalar: representações, práticas, interações e desafios [tese]. Rio de Janeiro: Escola Nacional de Saúde Pública da Fiocruz; 2000.

6. Farias MAA. Elaboração de trabalhos acadêmicos com formatação no Microsoft Word. Rondônia: SENAC; 2007.

7. Minayo MC. Violência e saúde. Rio de Janeiro: Fiocruz; 2006.

8. Ministério da Saúde (BR). Portaria MS/GM n 737, de 16 de maio de 2001.

Política Nacional de Redução da Morbimortalidade por Acidentes e Violências.
Brasilia: Diário Oficial da União; 18 maio 2001. Seção IE.

9. Deslandes S. Análise do discurso oficial sobre a humanização da assistência hospitalar. Ciênc Saúde Coletiva. 2004;9(1):7-14.

10. Biscaia A, Conceição C, Fronteira I, Craveiro I, Flores I, Santos O, et al. Violência no local de trabalho setor saúde: estudo de caso português. Relatório final. 2002. 11. Coler MA, Lopes $M$, Moreira M. Os profissionais de saúde frente à violência no idoso. Mudanças. 2008;16(2):116-22.

12. Vieira EM, Perdona GC, Almeida AM, Nakano AM, Santos MA, Daltoso D, et al. Conhecimento e atitude dos profissionais de saúde em relação à violência de gênero. Rev Bras Epidemiol. 2009;12(4):566-77.

13. Minayo MCS. Violência e saúde. Resenha de Montagner MA, Amorin RF, Silva JG, Lira SVG. Ciênc Saúde Coletiva. 2008;13(Suppl. 0).

14. Ministério da Saúde (BR). Impacto da violência na saúde dos brasileiros. Resenha de Morgado R. Ciênc Saúde Coletiva. 2006;11(2).

15. Altamirano RI. La violencia intrafamiliar: los mitos. Rev Med Guanajuato. 2000;10(1-2):206-9.

16. Masetto MT. Docência na universidade. Campinas: Papirus; 1998. 